\title{
Microfluidic channels laser-cut in thin double-sided tapes: cost-effective biocompatible fluidics in minutes from design to final integration with optical biochips
}

\author{
Daniel Patko $^{1,2}$, Zsolt Mártonfalvi ${ }^{3}$, Boglarka Kovacs ${ }^{1}$, \\ Ferenc Vonderviszt ${ }^{1,4}$, Miklós Kellermayer ${ }^{3,5}$ and Robert Horvath ${ }^{1 *}$ \\ ${ }^{I}$ Research Institute for Technical Physics and Material Science MFA Hungarian Academy of Sciences, \\ H-1120 Konkoly-Thege út 29-33, Budapest, Hungary \\ ${ }^{2}$ Doctoral School of Molecular- and Nanotechnologies, Faculty of Information Technology, \\ University of Pannonia; H-8200 Egyetem u.10, Veszprém, Hungary \\ ${ }^{3}$ Semmelweis University Department of Department of Biophysics and Radiation Biology H-1094 \\ Tüzoltó u. 37-47, Budapest, Hungary \\ ${ }^{4}$ Bio-Nanosystems Laboratory, Research Institute of Chemical and Process Engineering, Faculty of \\ Information Technology University of Pannonia; H-8200 Egyetem u.10, Veszprém, Hungary \\ ${ }_{5}$ MTA-SE Molecular Biophysics Research Group
}

*horvathr@mfa.kfki.hu

\begin{abstract}
A simple, reliable and cost-effective fluidic channel, fabricated by using double-sided pressure-sensitive tapes, is demonstrated here. A laser-cutting method is applied to engrave structures in sheets of the tapes. After peeling off the tape liners, the structures could be easily integrated at room temperature with label-free optical waveguide biochips without further modifications or additional processing steps. It is shown that the well-defined and controllable height of the channels is advantageous for stopped-flow measurements of analyte binding. The easy fabrication of a fully transparent integrated sensor unit - tape cuvette system is also demonstrated for parallel microscopic investigations. The transparent unit was used to on-line monitor the surface adhesion of Salmonella cells on poly-L-lysine-coated biochip surfaces, followed by the straightforward microscopic visualization of the adhered bacterial cells. The material of the double sided tape is stable in aqueous solutions. Furthermore, its material is biocompatible, making it ideal for biological applications. Excellent, stable and reversible bonding of the microstructured tapes to biocompatible plastic and glass is also demonstrated. The simplicity of the fabrication at ambient temperatures makes the developed processes appealing for lab-on-a-chip applications, particularly if the bonded biochips are precious.
\end{abstract}




\section{Introduction}

The need for reliable, cheap, disposable lab-on-a-chip and point-of-care devices is on the rise [1-5]. These systems are utilized in biotechnology, medical treatment and diagnosis, and in basic research as well [6,7]. A key issue in all of these developments is to find cost-effective and reliable methods for handling body fluids, aqueous solutions of biological molecules or living cells. Decreasing the sample volume is also critical. These needs initiated the development of microfluidic channels to handle minute quantities of liquid samples. Usually these channels are made of silicon, glass or polymers, and their fabrication and final integration involves costly and laboratory-intensive etching, baking or bonding steps [8]. An important step was the development of PDMS microfluidic channels which enabled rapid replication from a master $[8,9,10]$. However, even the fabrication of the master itself is cost intensive and usually requires a Clean Room. Moreover, the final integration of the PDMS channels involves plasma treatment or baking, making it difficult to integrate structures which are pre-coated with sensitive biological materials such as antibodies. It is also time-consuming to modify the channel design because it requires the fabrication of a new master structure.

In the present contribution we demonstrate the cheap, reliable and rapid fabrication of microfluidic channels in pressure-sensitive double-sided tapes. The applied tapes are available in various thicknesses and are biocompatible. Laser engraving was used to fabricate structures in the tapes. It is demonstrated that these novel cuvettes can be easily integrated with both polymers and glass. To demonstrate the integration and test the methodology, the cuvettes were integrated with planar optical waveguide-based sensor chips. The obtained sensing units were applied to measure the refractive index of aqueous solutions and follow the surface adsorption of proteins. A completely transparent configuration is also demonstrated. The welldefined thickness of the fabricated channels was exploited in stopped-flow measurements. The definitive advantages of the newly-developed fluidic channels are the remarkably short time and ease of their design, fabricatation and integration. Finally, the fabrication and integration steps can be carried out at ambient conditions, alleviating the need for difficult chemical etching, plasma treatment or baking procedures. 


\section{Materials and methods}

\subsection{Double-sided pressure-sensitive tapes}

ARcare ${ }^{\circledR} 8890$ and 90445 (Adhesives research Inc.) clear polyester double sided pressure sensitive tapes with MA-61 and As-110 acrylic medical grade adhesive coatings were used with total thicknesses of $50.8 \mu \mathrm{m}$ and $81.28 \mu \mathrm{m}$, respectively. The $12.7 \mu \mathrm{m}$ and $25,4 \mu \mathrm{m}$ thick tape substrates, as well as their adhesive coatings, are biocompatible and insoluble in aqueous solutions. At both faces of the tapes a $50.8 \mu \mathrm{m}$ thick polyester release liner is placed by the manufacturer. These liners were removed before final integration of the structured tapes. For dissolving the adhesive coatings and removing the tapes from glass and polyether ether ketone (PEEK) surfaces butanone (Methyl-Ethyl-Ketone or MEK, VWR International, 25654.292), was used.

\subsection{Structural design and laser cutting}

The design of the microstructures was first drawn in Corel Draw. The Corel files in vector mode were printed on an Epilog Mini 18 (25W) $\mathrm{CO}_{2}$ laser engraver and cutter device. The laser power was optimized and finally set to $5 \mathrm{~W}$ to effectively cut the tapes across with smooth edges. The lines in the Corel drawing defined the cutting path of the laser beam, therefore any modifications on the design was straightforward and easy.

\subsection{Optical waveguide sensor chips}

The fabricated fluidic channels in the double-sided types were integrated with planar optical waveguide-based sensor chips used for Grating Coupled Interferometry (GCI) and for Optical Waveguide Lightmode Spectroscopy (OWLS). The GCI sensor was a planar optical waveguide from Creoptix $\mathrm{GmbH}$ with dimensions $8 \times 12 \times 1 \mathrm{~mm}$. The chip consists of a 155$\mathrm{nm}$-thick $\mathrm{Ta}_{2} \mathrm{O}_{5}$ layer deposited on glass. The sensor had two in- and one outcoupling grating structures for transferring the GCI signal into a fiber-coupled detector [11]. The OWLS sensor chip (type OW2400, Microvacuum Ltd., Hungary) was a 16x48x0.5 mm glass substrate coated with a 176-nm-thick $\mathrm{TiO}_{2} \mathrm{SiO}_{2}$ sol-gel film. For mode excitation a 1-mm-wide grating was formed in the film at the center of the chips, in parallel with the shorter edges [12,13]. For interrogating the waveguide sensors and recording the effective refractive index changes of the waveguide modes a benchtop GCI setup [11] and a BIOS OWLS system (Microvacuum Ltd., Hungary) [14] were used. Before integration, the sensor chips were cleaned with 
chromsulfuric acid according to published protocols $[15,16]$ and dried with a stream of clean nitrogen gas.

\subsection{Assembling and sealing the fluidic channels}

After mounting the structured tapes onto the sensor chips, they were sealed by using two types of covering units. The first type was a machined plastic part made of PEEK. Separate units were made for the GCI and for the OWLS systems. The PEEK parts contained $0.5 \mathrm{~mm}$ i.d. drilled holes for inlet and outlet tubings. The second type was made of a glass plate with exactly the same dimensions as the GCI chip. Two holes with $1 \mathrm{~mm}$ i.d. were drilled through the glass to enable the connection of inlet and outlet Teflon tubings. The covering units were carefully cleaned prior to assembly.

\subsection{Liquid handling}

Teflon tubing with an i.d. of $0.5 \mathrm{~mm}$ was used in all of the experiments. The cuvettes were filled with the solution of interest by using an Ismatec peristaltic pump with 12 rollers. The 0.5-mm-i.d. flanged Tygon tubing positioned on the pump was connected to the Teflon tubing with linear connectors (Omnifit). To inject minute amounts of samples during stopped-flow OWLS experiments, a septum injection port was integrated in the inlet of the PEEK covering unit. A Hamilton syringe was used to inject the investigated solutions through the septum. 5\% glycerol in MQ water (refractive index 1.33676) or $10 \mu \mathrm{g} / \mathrm{mL}$ avidin (Sigma-Aldrich) in phosphate buffered saline (PBS, $\mathrm{pH}=7.4$, Sigma-Aldrich) were used for testing the devices and demonstrating their sensing capabilities. Salmonella typhimurium (SJW2536 Salmonella typhimurium) suspension was used in the bacterial adhesion tests. The cells were suspended in PBS (phosphate-buffered saline). The employed GCI chip was coated with poly-L-lysine (PLL) using PLL (Sigma-Aldrich) dissolved in MQ water at a final concentration of 0.1 $\mathrm{mg} / \mathrm{mL}$. 


\section{Results and discussions}

\subsection{Dual-channel tape cuvette: fabrication and integration with GCI sensor chips}

In the first experiment, a dual-channel microcuvette was fabricated for the GCI sensor chips. First, a 15x15-cm sheet of ARcare 8890 tape was placed into the laser cutting system. Here, a focused $\mathrm{CO}_{2}$ laser beam (focal length $2.54 \mathrm{~cm}$, spot size around 20-80 $\mu \mathrm{m}$ ) cuts across the tape at pre-defined locations by positioning the beam with a motorized mirror (see Fig. 1a).

(a)
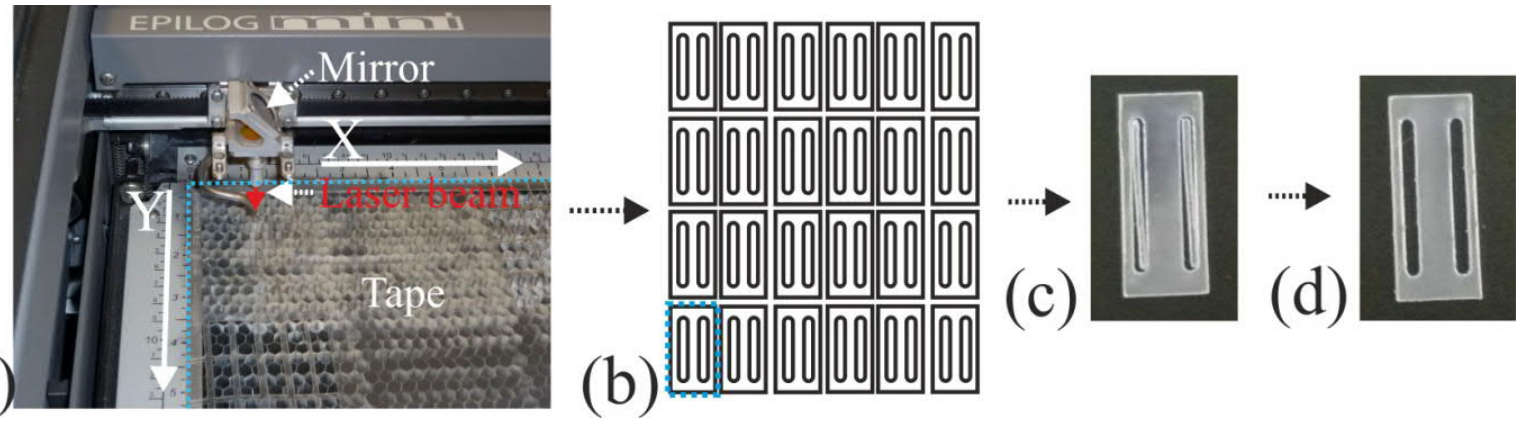

Figure 1. (a) The Epilog Mini laser engraver and cutter. The double sided tape as well as the mirror reflecting the cutting laser beam onto the tape is clearly seen. Motorized stages can move the mirror in $\mathrm{x}-\mathrm{y}$ directions to position the beam on the tape. (b) The design of the 24 structures cut out from the tape. (c-d) The final unit, with and without the cut parts inside the channels.

During the first demonstration, 24 pieces of dual-channel units were fabricated (the arrangements of the channels are shown in Fig. 1b). Subsequently, the structured tapes were taken out of the laser cutter, and the liners of the tapes were removed. Finally, the channels were placed onto the GCI sensor chip, and the plastic covering unit with inlet and outlet tubings were used to seal the channels (see Fig. 1b-d and Fig. 2a,b). For final bonding the entire assembly was pressed together by hand (see Fig 2a). Images of the assembled device are shown in Fig. 2b. Importantly, the entire collection of 24 structured tapes shown in Fig. 1b were processed in only about 2 minutes, and the final integration took about another minute. We note that the narrowest channel which could be fabricated using the ARcare ${ }^{\circledR}$ 8890 tape had a width of around $120 \mu \mathrm{m}$. Therefore, the developed technology is not applicable for high-precision nanofluidics, but could be a reasonable cost-effective solution in several applications, such as the fabrication of fluidic channels for cellular studies. 


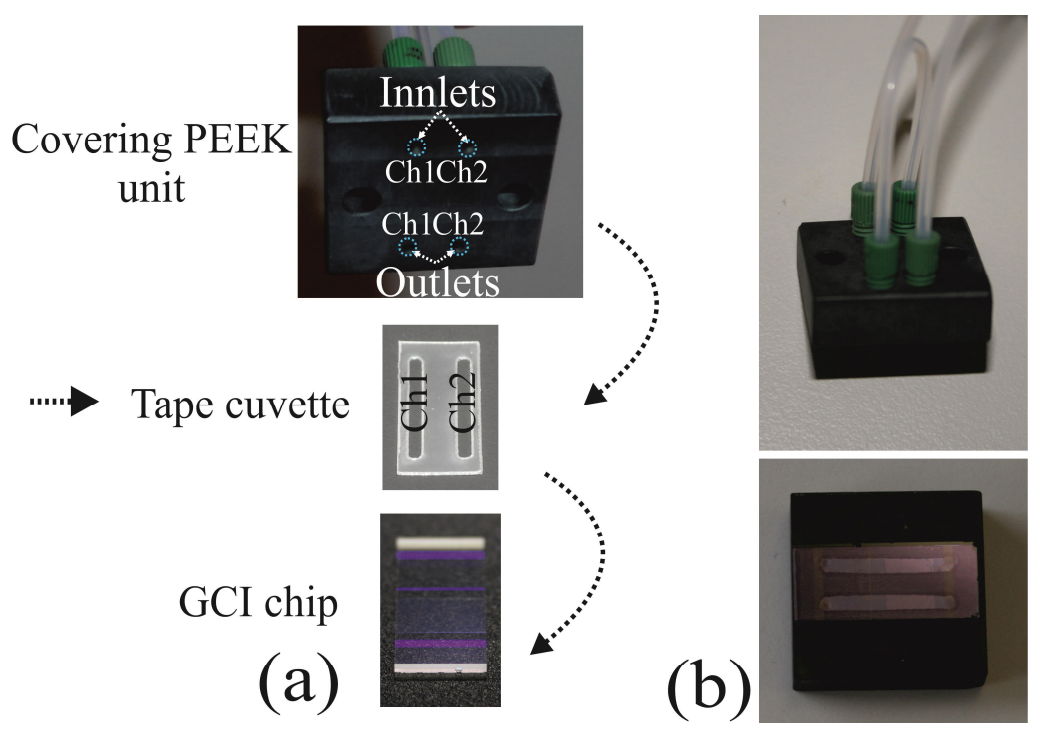

Fig 2. (a) The GCI sensor, structured double sided tape and PEEK covering unit before assembly. (b) Photographs of the assembled module. The two inlet and two outlet tubing are clearly visible.

The GCI sensor with integrated fluidics shown in Fig. 2b was then placed into an optical arrangement described in ref. [11]. Here the phase shifts of the waveguided mode travelling under the fluidic channels were recorded with a precision of $\sim 10^{-4} \mathrm{rad}$. In order to test the fabricated channels (see Fig. 3), MQ water was first injected into both channels, and the phase signals were recorded as a function of time. No leakage whatsoever was observed. After recording a stable baseline, $5 \%$ glycerol solution was injected into both channels by using the peristaltic pump. This resulted in a continuous increase in the recorded phases which saturated after the entire channels were filled with the glycerol solution. Subsequently, pure water was again injected into the channels. During sample solution change the pump was stopped for $3 \mathrm{~s}$. 


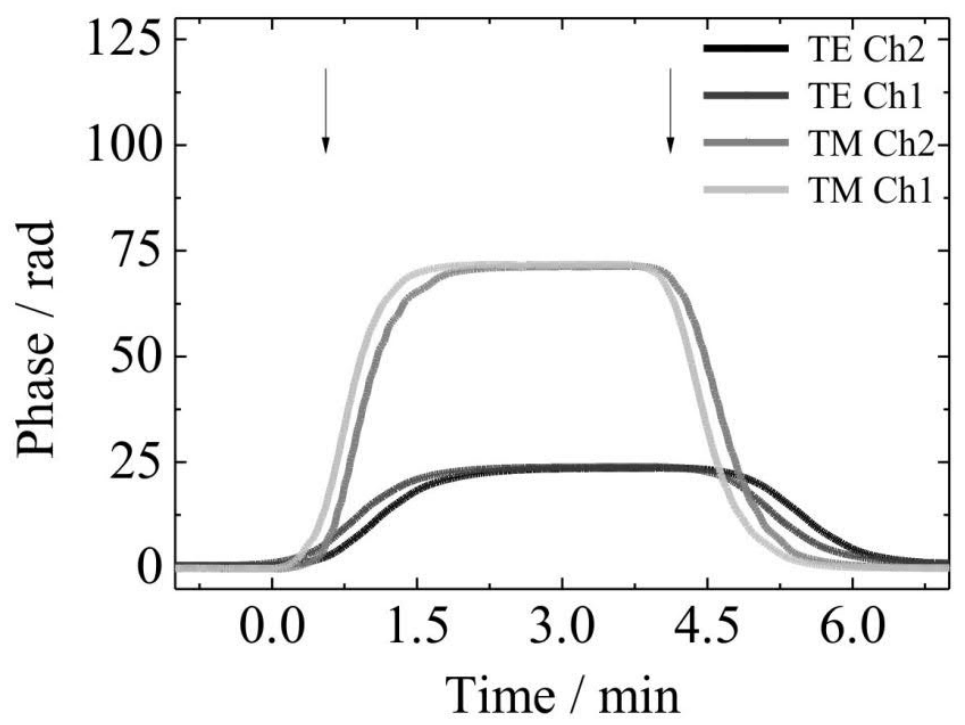

Fig. 3. Phase signals when $5 \%$ glycerol was injected into the channels and exchanged back to MQ water. The experiment was performed with both TE and TM polarized modes. The theoretically higher refractive index sensitivity of the TM mode is verified by the experiment.

The recorded phases decayed back to the baseline level. The experiment was performed with both TE and TM polarized light. Fig. 3 demonstrates that the GCI chip with TM polarization has approximatey three times greater refractive-index sensitivity than the chip using TEpolarized modes [11]. It is important to stress that the recorded phase shifts were identical in both channels demonstrating the excellent lateral homogeneity of the GCI sensor chip and the feasibility of using one of the channels as reference. Furthermore, no drift was observed in the recorded phase signals; thus, there was no material leakage from the tapes. Notably, the tape can be removed with butanone, and after standard cleaning processes both the optical chip and cuvette covering unit can be re-used for further integrations and experiments.

\subsection{Single-channel tape cuvette for stopped-flow experiments using OWLS}

In the second experiment, a single-channel tape cuvette was fabricated for OWLS. The double-sided tape \#90442 was used in these experiments. A channel with dimensions 9x1.5 mm was cut in the centre of a circle-shaped piece (see Fig. 4a). After removing the liners, the tape was placed in the center of an OWLS sensor chip. Subsequently, a PEEK covering unit was pressed on the top of the tape to seal the channel and position the inlets and outlets at its ends. A septum injection port was integrated at the inlet. The different parts prior to assembly are shown in Fig. 4a. The assembled device was placed into a BIOS OWLS scanner, where the effective refractive indices of the modes supported by the waveguide sensor were recorded. 
First, PBS buffer was injected into the cuvette and a baseline signal was recorded. After about 5 minutes, $50 \mu \mathrm{L}$ of avidin solution was injected into the cuvette with a Hamilton syringe. This led to a rapid increase in the OWLS signal which saturated after about 10 minutes. The recorded effective refractive indices can be used to calculate the surface-adsorbed protein mass $[6,12]$. The results are shown in Fig. 4b. After the first injection the adsorbed protein mass saturated at a level of $0.19 \mu \mathrm{g} / \mathrm{cm}^{2}$. This value is comparable to the total protein amount present inside the cuvette. Therefore, one can expect that a dynamic equilibrium is reached when the protein concentration increased at the surface and decreased in the bulk. This assumption was verified with a second injection. Another bolus of $50 \mu \mathrm{L}$ of avidin solution was injected into the cuvette, increasing the bulk protein concentration to the initial level, thereby forcing further proteins to be adsorbed. This adsorption-injection cycle was repeated six more times. Following the seventh injection the adsorbed protein mass did not increase singificantly. Thus, the surface reached its maximum coverage at this bulk concentration.

The above type of experiment, in which the adsorption equilibrium is reached with a limited sample amount in successive injection steps is known as the stopped-flow method [17]. Our results demonstrate the applicability of the tape channels for stopped-flow experiments and pave the way for more sophisticated binding experiments.
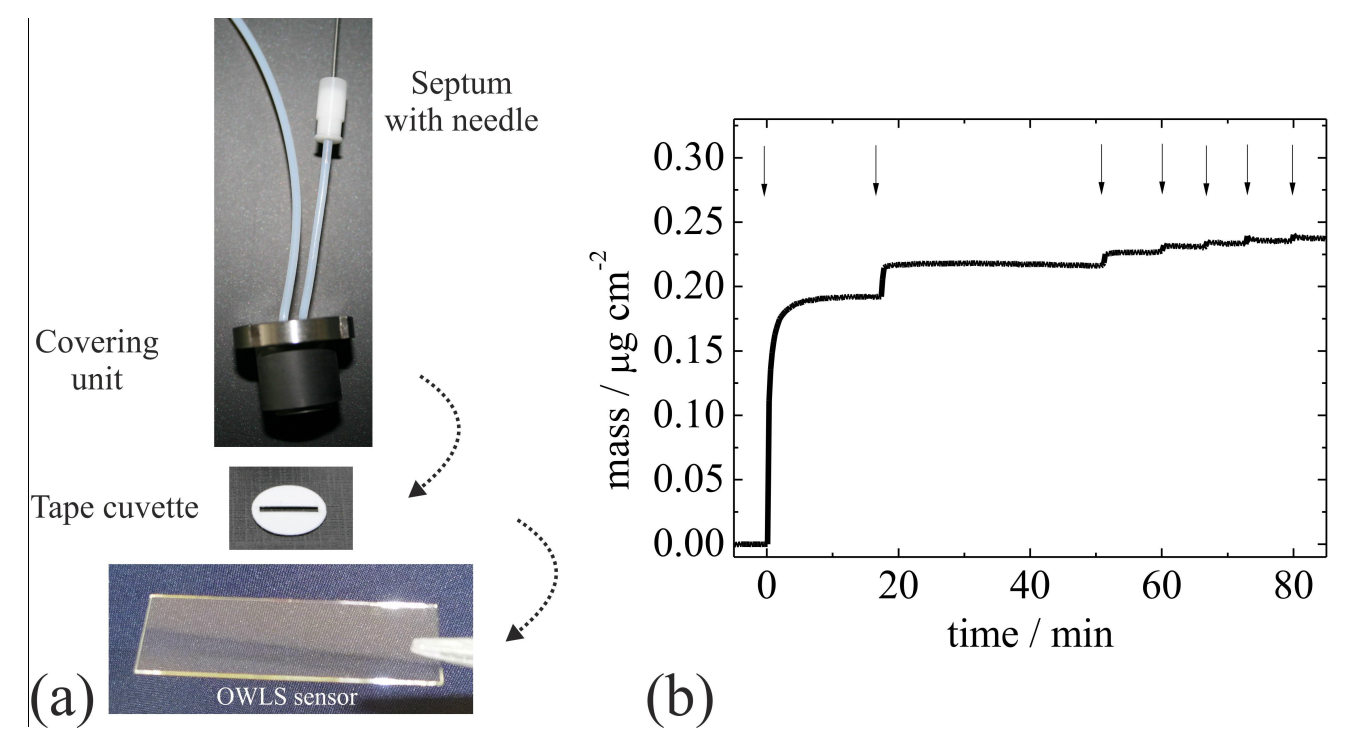

(b)

Fig. 4. (a) Components of the OWLS cuvette system prior to assembly. (b) Stopped-flow experiment using the assembled sensor unit..Arrows indicate the moments of injection of avidin solution into the channel with a Hamilton syringe. Maximum surface coverage is reached by seven successive injections of the same avidin solution. 


\subsection{Fully transparent integrated system using a covering glass plate with drilled holes - application for bacterial adhesion studies}

In the previous experiments the unit covering and sealing the tape channels was made of a non-transparent biocompatible polymer. Lack of transparency limits applicability, because in many cases it is desirable to investigate the sensor surface by using various modes of optical microscopy. Here we developed a fully transparent cuvette assembly for GCI. A glass covering unit was used with holes drilled in the glass for fixing the inlet and outlet tubings (see Fig. 5a). The Teflon tubing was narrowed by longitudinal stretching. The narrow end of the tube was then inserted into the inlet (or outlet) and positioned appropriately. Overextending pieces of the tube were removed with a scalpel. The assembled sensor-cuvette system is shown in Fig. 5b. The single fluidic channel and the coupling gratings of the GCI chip are clearly visible.
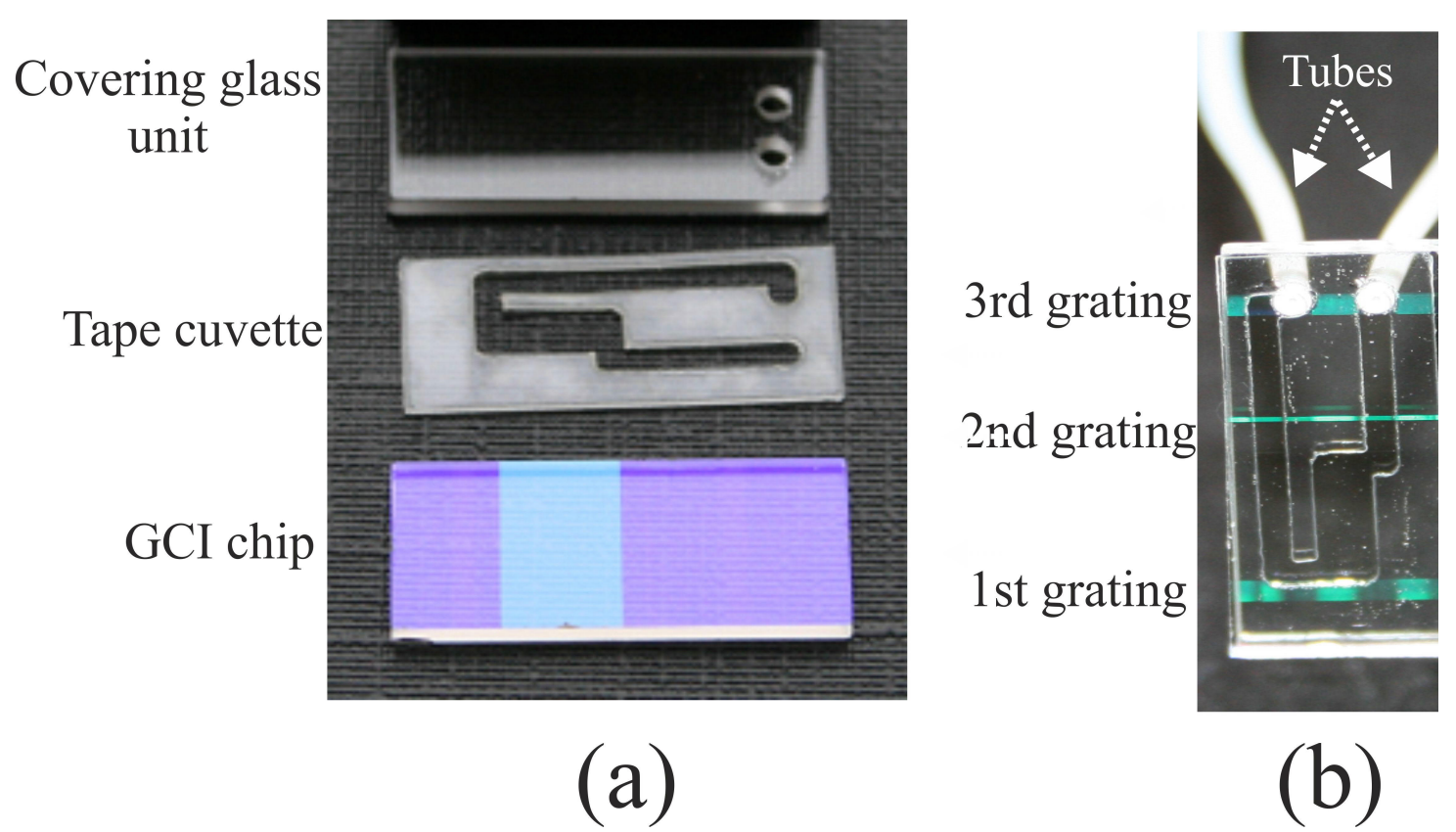

Fig. 5. (a) Components of the fully transparent system prior to assembly (GCI chip, tape cuvette, glass substrate with the two holes). (b) The demonstated fully transparent GCI device.

In order to demonstrate the applicability of the fully transparent integrated, unit a bacterial adhesion test was conducted using the GCI setup (see Fig. 6). First, the optical chip was coated with PLL and a baseline signal was recorded under PBS. After recording a stable baseline, $50 \mu \mathrm{l}$ bacterial solution was injected into the fluidic channel using a Hamilton syringe. The adhering bacterial cells increased the phase signal, which was saturated after 
about 3 minutes (see Fig. 6a). After saturation, $50 \mu 1$ PBS solution was injected to remove the reversibly adhering cells. Then, the whole transparent unit was put under a Zeiss Axiovert microscope to visualize the sensing surface. The adhered Salmonella cells were clearly visible (see Fig. 6b).

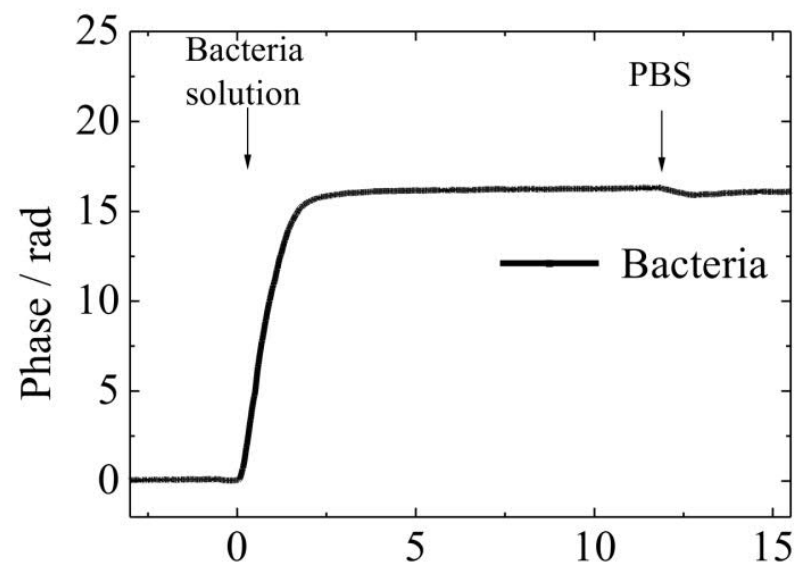

(a)

Time / $\min$

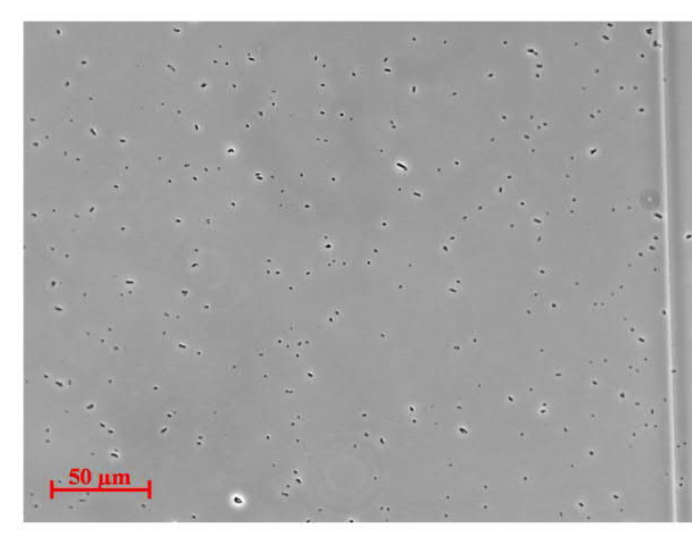

(b)

Fig. 6. (a) GCI phase shift signal during bacterial adsorption and desorption.. (b) Microscope image of the sensor surface after the GCI experiment. The adhered bacterial cells are clearly seen. The edge of the $\mathrm{SiO}_{2}$ layer is also visible at the right hand side of the image.

The demonstrated fully transparent arrangement can find further applications in parallel morphological investigations such as different modes of conventional light microscopy, Raman and infrared microscopies and spectroscopies.

\section{Conclusions}

The design, assembly and applicability of a simple, cheap and biocompatible microcuvette system based on ARcare ${ }^{\circledR}$ pressure sensitive double sided tapes was demonstrated in the present work. Channel structures in the tapes were cut with a laser engraver and cutter device. These types of biocompatible tapes are commercially available in various pre-defined thicknesses. The fabricated structured tapes were integrated with GCI and OWLS optical waveguide sensor chips, and the assembled units were tested by using various solutions. Stopped-flow measurement of protein adsorption and a fully transparent integrated device were also demonstrated using proteins and living bacterial cells. The developed tape cuvettes can find applications where rapid design and integration are priorities. A further advantage is that the integration of the channels does not require any plasma, heat or additional chemical 
treatment. Therefore, sensing units with pre-coated biological materials could be easily integrated with the proposed fluidic solutions. Future development may involve the application of double sided tapes with thinner liners in order to fabricate narrower channels.

\section{Acknowledgements}

This research was realized in the frames of TÁMOP 4.2.4. A/2-11-1-2012-0001 „National Excellence Program - Elaborating and operating an inland student and researcher personal support system convergence program" The project was subsidized by the European Union and co-financed by the European Social Fund. This work was supported by grants from the Hungarian Science Foundation (OTKA PD 73084 to RH and K84133 to MK), the Lendület program of the Hungarian Academy of Sciences and by the Hungarian National Development Agency grant TÁMOP-4.2.2/B-10/1-2010-0025. The continuous support of Creoptix AG (Switzerland) is gratefully acknowledged.

\section{References}

1. Y. Fang, "Label-free receptor assays," Drug Discov. Today Technol., 2010, 1, 5-11.

2. H. K. Hunt and A. M. Armani, "Label-free biological and chemical sensors," Nanoscale, 2010, 2, 1544-1559.

3. Y. Fang, A. M. Ferrie, N. H. Fontaine, J. Mauro, and J. Balakhrisnan, "Resonant waveguide grating biosensor for living cell sensing,” Biophys. J., 2006, 91, 1925-1940.

4. J. Homola, S. S. Yee, and G. Gauglitz, "Surface plasmon resonance sensors: review," Sens. Actuators.B Chem., 2009, 54, 3-15.

5. M.-Carmen Estevez, Mar Alvarez and Laura M. Lechuga, „Integrated optical devices for lab-on-a-chip biosensing applications” Laser Photonics Rev., 2012, 6, 463-487.

6. J. J. Ramsden, Biomedical Surfaces, Artech House, 2007.

7. M. Malmsten Biopolymers at Interfaces Taylor \& Francis, 2003.

8. S. K. Mitra, S. Chakraborty, Microfluidics and Nanofluidics Handbook, CRC Press Taylor and Francis, 2012.

9. J. D. Wang, Nicholas J. Douville , Shuichi Takayama and Mohamed ElSayed (2012) Quantitative Analysis of Molecular Absorption into PDMS Microfluidic Channels Annals of Biomedical Engineering, 2012, 40 1862-1873. 
10. V. Chokkalingam, B. Weidenhof, M. Krämer, W. F. Maier, S. Herminghaus and R. Seemann, Optimized droplet-based microfluidics scheme for sol-gel reactions Lab Chip, 2010, 10 1700-1705.

11. D. Patko, K. Cottier, A. Hamori, and R. Horvath, Single beam grating coupled interferometry: high resolution miniaturized label-free sensor for plate based parallel screening, Optics Express, 2012, 20, 23162-23173.

12. J. Voros, J. J. Ramsden, G. Csucs, I. Szendro, S. M. De Paul, M. Textor, and N. D. Spencer Optical grating coupler biosensors, Biomaterials, 2002, 23, 3699-3710.

13. W. Lukosz, Integrated optical chemical and direct biochemical sensors, Sens. and Actuators B., 1995, 29 37-50.

14. Web page of Microvacuum LtD, www.microvacuum.com

15. R. Horvath, H. C. Pedersen, N. Skivesen, D. Selmeczi, and N. B. Larsen, Monitoring of living cell attachment and spreading using reverse symmetry waveguide sensing Appl. Phys. Lett., 2005, 86, 071101.

16. S. Kurunczi, A. Hainard, K. Juhasz, D. Patko, N. Orgovan, N. Turck, J. C. Sanchez and R. Horvath, Polyethylene imine-based receptor immobilization for label free bioassays Sensors and Actuators B., 2013, 180, 71-76.

17. A Lionello, J. josserand, H. Jensen and H. H. Girault Dynamic protein adsorption in microchannels by "stop-flow" and continuous flow La bon a Chip, 2005, 5, 1096-1103. 